\title{
Goeckerman therapy versus methotrexate for psoriasis: a study on military personnel
}

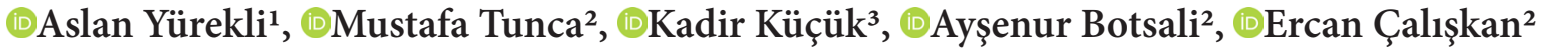 \\ ${ }^{1}$ Kuşadası Public Hospital, Dermatology Clinic, Aydın, Turkey \\ ${ }^{2}$ Gülhane School of Medicine, Department of Dermatology, Ankara, Turkey \\ ${ }^{3}$ Hitit University, Faculty of Medicine, Department of Dermatology Çorum, Turkey
}

Cite this article as: Yürekli A, Tunca M, Küçük K, Bostali A, Çalışkan E. Goeckerman therapy versus methotrexate for psoriasis: a study on military personnel. J Health Sci Med 2022; 5(1): 297-300.

\begin{abstract}
Introduction: Although methotrexate is a widely used systemic medication for psoriasis, a restrictive cumulative dosage limits its use in each patient, and a liver biopsy is recommended when the cumulative dose reaches 3.5-4.0 mg. As an alternative, Goeckerman therapy is a safe, efficient, tar-based method of treating psoriasis but one used increasingly less in recent decades due to the inconvenience of tar application and of requiring outpatients to remain covered in tar before receiving phototherapy hours if not a day later. However, for patients such as soldiers who can be treated as inpatients, Goeckerman therapy is preferable due to its efficacy and good safety profile.

Material and Method: We retrospectively evaluated 96 patients with psoriasis, all military personnel, who had been treated with either methotrexate $(n=49)$ or Goeckerman therapy $(n=47)$ between 2012 and 2016. Their baseline and exit Psoriasis Area and Severity Index (PASI) scores were comparatively analyzed.

Results: No statistical difference in relative recovery emerged between patients who had received methotrexate and ones who had undergone Goeckerman therapy. Both groups had achieved a mean PASI score of 75 at approximately the same time.

Conclusion: When the rapid return to work is important, we recommend using Goeckerman therapy to treat psoriasis given its relatively low side effect profile and lack of immunosuppressive action. Both advantages can benefit patients such as soldiers who are able to undergo treatment in inpatient settings, cannot meet physicians frequently due to work requirements, and cannot avoid the risk of infection (i.e., a risk factor for methotrexate use) due to living in crowded spaces.
\end{abstract}

Keywords: Goeckerman therapy, methotrexate, psoriasis vulgaris, coal tar, phototherapy

\section{INTRODUCTION}

Psoriasis is a chronic, multifactorial, inflammatory disease that affects an estimated $2-3 \%$ of the world's population. Although psoriasis can emerge at any age, the age of onset shows a bimodal peak at 20-30 and 50-60 years, and environmental, genetic, and immunological factors appear to play a role in its development (1). As for its pathophysiology, the activation and migration of $\mathrm{T}$ cells to the dermis triggers the release of cytokines, which causes inflammation and the rapid production of keratinocytes (2). Various treatment options are available for psoriasis, including topical agents, systemic agents, and phototherapy (3).

The analog of folic acid, methotrexate is used as an antineoplastic agent and to treat inflammatory disorders such as psoriasis as well as dermatomyositis, lupus erythematosus, sarcoidosis, and systemic sclerosis
(4). As an inhibitor of dihydrofolate reductase, methotrexate also inhibits the synthesis of folate $(5,6)$. Methotrexate was approved for the treatment of severe, recalcitrant, disabling psoriasis by the U.S. Food and Drug Administration in 1972. Although its maximum effects are generally achieved within 5 to 6 months, methotrexate usually shows some benefit within 6 to 8 weeks in response to skin diseases (4).

Goeckerman therapy can also be used to treat moderate to severe plaque psoriasis. First formulated in 1925 by William $\mathrm{H}$. Goeckerman, the therapy involves the combined application of crude coal tar and broadband ultraviolet-B (UVB), although narrow-band UVB may be used instead (7). Although the tar has to remain on the skin for at least 2 hours, clinic practice recommends extending the application overnight and thus applying the 
tar at bedtime, which also precludes the inconvenience of the smell and staining during the day (8).

Because the patient has to wait covered in tar for some time and because the application of tar stinks, stains clothes, causes mechanical discomfort, and thus reduces treatment compliance, Goeckerman therapy is not preferred in practice. However, in terms of efficacy and reliability, the therapy is indispensable for clinicians and may thus be preferable over other treatments for patients who can be treated in inpatient settings.

\section{MATERIAL AND METHOD}

The study was carried out with the permission of Gülhane School of Medicine Non-Interventional Clinical Researchs Ethics Committee (Date: 08.03.2016, Decision No: 3-117). All procedures were carried out in accordance with the ethical rules and the principles of the Declaration of Helsinki. After a local ethics committee approved our study, we evaluated the data of patients with psoriasis who were treated at our clinic with methotrexate or Goeckerman therapy between 2012 and 2016. Clinical outcomes were obtained by screening outpatient and clinical records. Once patients without complete data in their medical records were screened out, 96 patients remained and were included in our sample. Whereas 49 of them had received methotrexate treatment, the 47 others had undergone Goeckerman therapy. All of the patients were military personnel, and none had received any adjuvant therapy.

In the group of patients who had received Goeckerman therapy (i.e., Goeckerman group), a mixture containing $5 \%$ coal tar and $2 \%$ salicylic acid had been applied to the entire body, left for 10 hours overnight, and removed in the morning with liquid petroleum jelly. Once the patients had bathed, they proceeded to the phototherapy unit to receive broadband UVB phototherapy. The initial dose to be administered to each patient had been determined according to their Fitzpatrick skin type. The patients underwent the entire procedure described above for five days, during which erythema responses were assessed on a daily basis and doses adjusted accordingly.

In the group of patients who had been treated with methotrexate (i.e., methotrexate group), $10-25 \mathrm{mg}$ of methotrexate per week had been subcutaneously administered to each patient for six weeks. To reduce the frequency of side effects, each patient had also received $5 \mathrm{mg}$ of folic acid every day except on the day of methotrexate administration.

We retrospectively assessed the efficacy of the treatments in light of baseline and exit Psoriasis Area and Severity Index (PASI) scores, and each patient's time to reach a PASI score of 75 was determined.
Statistical analysis was performed using SPSS 15.0 for Windows (SPSS Inc., Chicago, IL, USA). The MannWhitney U test was used to analyze the age, baseline PASI score, and time to reach a PASI score of 75 in each group.

\section{RESULTS}

All patients were male. In the Goeckerman group, the mean age of patients was $26.1 \pm 6.7$ years, and the baseline PASI score $(\mathrm{M} \pm \mathrm{SD}$ ) was $14.1 \pm 2.6$ (Table 1). By little contrast, the mean age in the methotrexate group was $26.7 \pm 5.2$ years, and the baseline PASI score was $14.3 \pm 2.3$. Thus, as shown in Table 2, no statistically significant difference emerged between the treatment groups in terms of age $(p>.355)$ or baseline PASI score (p>.464). Likewise, the time to achieve a PASI score of 75-26.1 \pm 5.08 and 25.8 \pm 5.95 days in Goeckerman and methotrexate groups, respectively-did not significantly differ between the groups ( $>$.790) (Table 2).

\begin{tabular}{|lcc|}
\hline \multicolumn{3}{|l|}{ Table 1. Characteristics of patients } \\
\hline Treatment & Age in years & Initial PASI score \\
\hline Goeckerman therapy & $26.1 \pm 6.7$ & $14.1 \pm 2.6$ \\
Methotrexate & $26.7 \pm 5.2$ & $14.3 \pm 2.3$ \\
p & $>.05$ & $>.05$ \\
\hline
\end{tabular}

\begin{tabular}{|lcl|}
\hline \multicolumn{2}{|l|}{ Table 2. Response to treatment (day) } & \\
\hline Treatment & Response to treatment (day) & \\
\hline Goeckerman therapy & $26.1 \pm 5.08$ & $\mathrm{p}>.05$ \\
Methotrexate & $25.8 \pm 5.95$ & $\mathrm{p}>.05$ \\
\hline
\end{tabular}

\section{DISCUSSION}

Topical agents play an important role in treating psoriasis and can be combined with all treatment options. Such agents modify the permeability of skin lesions to increase the transmission of UV rays and thus improve therapeutic efficacy. Among such agents, topical tar has been used to treat dermatological conditions for years, especially as part of Goeckerman therapy, which is as effective as other treatments in treating psoriasis and relatively safe (9). Both the tar and UVB used in the therapy have been hypothesized to inhibit the hyperproliferation of keratinocytes, modulate inflammatory cytokines, and deplete $\mathrm{T}$ lymphocytes when used together, all for an increased cumulative effect (10). Regarding remission, the therapy has also been more successful than other phototherapy regimens.

Goeckerman therapy originally consisted of soaking in crude coal tar day and night, followed by gradually increasing exposure to UV radiation after the tar was removed. Although the tar should stay on the body for at least 2 hours, the longer the application, the better the results (9). Side effects of the topical application of 
raw coal tar include tar folliculitis, acneiform sputum, contact dermatitis, acute tar toxicity and atrophy, telangiectasia, pigmentation, exfoliative dermatitis, and, far more rarely, keratoacanthomas (10). In regard to the remission periods, Goeckerman therapy is proven to be more succesful than the other phototherapy regimens.

The most concerning side effect of Goeckerman therapy is the increased risk of skin cancer. Despite the proven carcinogenic effects of PUVA, those effects remain controversial when UVB is used. In a study with 1,373 patients, Stern et al. (11) found that patients who had received recurrent Goeckerman therapy showed an increase in skin cancer. By contrast, Studniberg et al. (12) have reported that available data indicate that therapeutic UVB poses a low risk of producing cutaneous cancers, with the possible exception of producing ones on male genital skin, whereas oral PUVA poses a definite cutaneous carcinogenic risk. In the same vein, Hearn et al. $(13,14)$ observed no significant association between NB-UVB treatment and BCC, SCC, or melanoma, despite a small increase in $\mathrm{BCC}$ among patients treated with PUVA.

Methotrexate, formerly known as amethopterin, is a chemotherapeutic agent that affects the immune system by competitively inhibiting dihydrofolate reductase, an enzyme involved in the synthesis of tetrahydrofolate. Methotrexate's efficacy against psoriasis is apparent from its antiproliferative and anti-inflammatory effects (4). The dose may be gradually increased until the clinical response is achieved but should not exceed $30 \mathrm{mg} / \mathrm{week}$, and treatment should be continued with the lowest dose that achieves remission. To reduce the frequency of side effects, oral folic acid supplementation of 1-5 mg should also be given daily except on the day of methotrexate administration $(4,5)$. Before treatment, the patient should receive a physical examination and their medical history should be comprehensively evaluated, especially for the likelihood of alcohol intake, exposure to hepatitis B or $\mathrm{C}$, and familial liver disease. Laboratory tests, including a $\mathrm{CBC}$ with differential, creatinine, and liver function tests for albumin and bilirubin, should be obtained for baseline levels, and a purified protein derivative test or another screening test for latent tuberculosis should also be conducted at baseline, particularly if the patient's history indicates risk. When risk factors are absent, a liver biopsy should be performed when the cumulative dose of methotrexate reaches 3.5-4.0 g. If risk factors such as alcoholism, a high level of liver enzymes, and obesity are present, however, then a liver biopsy should be performed when the cumulative dose reaches 1.5-2.0 $\mathrm{g}$ (15-17). The most common adverse effects of methotrexate include hepatotoxicity, ulcerative stomatitis, leukopenia and, thus predisposition to infection, nausea, abdominal pain, fatigue, fever, dizziness, acute pneumonitis, and, more rarely, pulmonary fibrosis and kidney failure. Because methotrexate is teratogenic, it is not used in pregnancy (18-20).

In our retrospective study, conducted on a subpopulation of military personnel-that is, individuals who cannot be required to visit physicians frequently due to their workrelated responsibilities and who cannot avoid the risk of infection because they live in crowded spaces-patients had been treated with either Goeckerman therapy or systemic treatment for a similar period of time. We examined the use of Goeckerman therapy given its advantages for return to work as a method that does not require follow-up after hospitalization. In treatment with methotrexate, however, patients have to be followed up during the treatment period and in the weeks that follow and cannot be present in crowded workplaces due to the risk of infection.

Because we examined a subpopulation available for treatment requiring hospitalization and for whom it is important to avoid the side effects of methotrexate treatment, whether the effectiveness of Goeckerman therapy is similar to that of systemic therapy in other specific populations should be examined in future studies.

\section{CONCLUSION}

Although Goeckerman therapy is a largely forgotten treatment, it remains effective and reliable for treating psoriasis. In our study, we found similar effects between Goeckerman therapy and methotrexate. However, given its lower side effect profile, Goeckerman therapy should be used more widely among patients such as military personnel who can be treated as inpatients.

\section{ETHICAL DECLARATIONS}

Ethics Committee Approval: The study was carried out with the permission of Gülhane School of Medicine Non-Interventional Clinical Researchs Ethics Committee (Date: 08.03.2016, Decision No: 3-117).

Informed Consent: Because the study was designed retrospectively, no written informed consent form was obtained from patients

Referee Evaluation Process: Externally peer-reviewed.

Conflict of Interest Statement: The authors have no conflicts of interest to declare.

Financial Disclosure: The authors declared that this study has received no financial support.

Author Contributions: All of the authors declare that they have all participated in the design, execution, and analysis of the paper and that they have approved the final version. 


\section{REFERENCES}

1. Michalek IM, Loring B, John SM. A systematic review of worldwide epidemiology of psoriasis. J Eur Acad Dermatol Venereol 2017; 31: 205-12.

2. Ferreira BI, Abreu JL, Reis JP, et al. Psoriasis and associated psychiatric disorders: a systematic review on etiopathogenesis and clinical correlation. J Clin Aesthet Dermatol 2016; 9: 36-43.

3. Kelly JB, Foley P, Strober B. Current and future oral systemic therapies for psoriasis. Dermatol Clin 2015; 33: 91-109.

4. Diani M, Grasso V, Altomare G. Methotrexate: practical use in dermatology. G Ital Dermatol Venereol 2016; 151: 535-43.

5. Haustein UF, Rytter M. Methotrexate in psoriasis: 26 years' experience with low-dose long-term treatment. J Eur Acad Dermatol Venereol 2000; 14: 382-8.

6. Strober BE, Siu K, Menon K. Conventional systemic agents for psoriasis: a systematic review. J Rheumatol 2006; 33: 1442-6.

7. Gupta R, Debbaneh M, Butler D, et al. The Goeckerman regimen for the treatment of moderate to severe psoriasis. J Vis Exp 2013; 77: Article 50509.

8. Lee E, Koo J. Modern modified "ultra" Goeckerman therapy: a PASI assessment of a very effective therapy for psoriasis resistant to both prebiologic and biologic therapies. Journal of Dermatological Treatment 2005;16: 102-7.

9. Çalıskan E, Tunca M, Açıkgöz G, et al. Narrow band ultraviolet-B versus Goeckerman therapy for psoriasis with and without acitretin: a retrospective study. Indian J Dermatol Venereol Leprol 2015; 81: 584-7.

10.Zhu TH, Nakamura M, Farahnik B, et al. The Patient's Guide to Psoriasis Treatment. Part 4: Goeckerman therapy. Dermatol Ther (Heidelb) 2016; 6: 333-9.

11.Stern RS, Zierler S, Parrish JA. Skin carcinoma in patients with psoriasis treated with topical tar and artificial ultraviolet radiation. Lancet 1980; 2: 732-3.

12.Studniberg HM, Weller P. PUVA, UVB, psoriasis, and nonmelanoma skin cancer. J Am Acad Dermatol 1993; 29: 1013-22.

13. Hearn RM, Kerr AC, Rahim KF, et al. Incidence of skin cancers in 3867 patients treated with narrow-band ultraviolet B phototherapy. Br J Dermatol 2008; 159: 931-5.

14.Şentürk N. Methotrexate. Turkderm-Arch Turk Dermatol Venerology 2016; 50: 18-21.

15.Hugh JM, Weinberg JM. Update on the pathophysiology of psoriasis. Cutis 2018; 102: 6-12.

16. Korman NJ. Management of psoriasis as a systemic disease: what is the evidence? Br J Dermatol 2020; 182: 840-8.

17.Schleicher SM. Psoriasis: Pathogenesis, Assessment, and Therapeutic Update. Clin Podiatr Med Surg 2016; 33: 355-66.

18. Alwan W, Nestle FO. Pathogenesis and treatment of psoriasis: exploiting pathophysiological pathways for precision medicine. Clin Exp Rheumatol 2015; 33: 2-6.

19. Griffiths CEM, Armstrong AW, Gudjonsson JE, Barker JNWN. Psoriasis. Lancet 2021; 397: 1301-15.

20.Staubach P, Zimmer S. Plaque psoriasis - more than a skin disorder. Med Monatsschr Pharm 2017; 40: 231-3. 